02

\title{
Оптические свойства и электронные характеристики празеодима и неодима в конденсированном состоянии
}

\author{
(ㄱ Л.А. Акашев, Н.А. Попов , В.Г. Шевченко \\ Институт химии твердого тела Уральского отделения РАН, \\ 620990 Екатеринбург, Россия \\ ฯ e-mail: n168@mail.ru \\ Поступила в редакцию 16.11.2020 г. \\ В окончательной редакции 04.03.2021 г. \\ Принята к публикации 23.03.2021 г.
}

\begin{abstract}
Исследованы оптические свойства неокисленной поверхности поликристаллических пленок празеодима и неодима в спектральном диапазоне 0.4-2.6 $\mu \mathrm{m}$. Оптические постоянные измерены эллипсометрическим методом Битти. Из полученных оптических постоянных рассчитаны дисперсионные зависимости световой проводимости $\sigma$, отражательной способности $R$, мнимой и действительной частей диэлектрической проницаемости $\varepsilon_{1}$ и $\varepsilon_{2}$, функции характеристических потерь энергии электронов $\operatorname{Im}(\varepsilon)^{-1}$. Представлены оптические свойства как пленок, так и жидких металлов, исследованных ранее. С использованием результатов измерений в инфракрасной области спектра по модели двухполосной проводимости рассчитаны электронные характеристики празеодима и неодима в твердом тонкопленочном и жидком состояниях.
\end{abstract}

Ключевые слова: эллипсометрия, оптические свойства твердых и жидких редкоземельных металлов, празеодим, неодим, характеристики электронов проводимости.

DOI: $10.21883 / \mathrm{OS} .2021 .07 .51075 .1614-21$

\section{Введение}

Тонкие пленки редкоземельных металлов (РЗМ), привлекают внимание исследователей в совершенно разных областях науки и техники. Металлические пленки РЗМ изучаются с точки зрения их магнитных свойств [1], в качестве основы для гетероструктур. РЗМ в тонкопленочном виде как смесь изомеров (долгоживущих ядерных состояний) также рассматриваются в качестве источников гамма-излучения и ядерной энергии [2,3]. На пленках РЗМ, полученных методами вакуумного напыления, проводят гидрирование [4-6], окисление [7,8] и другие исследования [9]. Оптические свойства РЗМ в твердом металлическом состоянии изучаются с конца прошлого века как с помощью численных расчетов [10], так и экспериментально [11-21]. Тонкопленочные Prи Nd-образцы изучались авторами $[16,18-20]$ в ультрафиолетовой и высокоэнергетической областях спектра. Оптические исследования на пленках $\operatorname{Pr}$ и $\mathrm{Nd}$ (в диапазоне 200-800 nm) проводились лишь в работах [22,23]. В [22,23] очень тонкие (от 3 до $27 \mathrm{~nm}$ ) пленки $\operatorname{Pr}$ и $\mathrm{Nd}$ изучались методом спектрофотометрии на пропускание. Были получены спектры поглощения $(\alpha)$, пропускания $(T, \%)$ и отражения $(R, \%)$, спектральные максимумы при этом значительно зависели от толщины металла. Значительно большую информативность в изучении оптических характеристик тонких пленок имеет метод эллипсометрии. В настоящей работе, кроме спектральных зависимостей коэффициентов отражения и поглощения поликристаллических пленок празеодима и неодима, представлены спектры показателей преломления $n$, коэффициентов поглощения $k$, световой проводимости $\sigma$, функции характеристических потерь $\operatorname{Im}(\varepsilon)^{-1}$, действительной $\left(\varepsilon_{1}\right)$ и мнимой $\left(\varepsilon_{2}\right)$ частей диэлектрической проницаемости. Проведено сравнение указанных характеристик для пленок и для жидких металлов $\operatorname{Pr}$ и $\mathrm{Nd}$, измеренных тем же методом в работах [24-26]. С использованием результатов измерений в инфракрасной (ИК) области спектра по модели двухполосной проводимости рассчитаны электронные характеристики указанных металлов в твердом и жидком состояниях.

\section{Методика эксперимента}

Оптические постоянные металлов в твердом и жидком состояниях измерялись эллипсометрическим методом Битти (эллипсометр ЛЭФ-3М, спектральный диапазон 0.4-2.6 $\mu \mathrm{m}$, угол падения $\varphi=82^{\circ}$ ) [27]. Установка, на которой изучались оптические свойства жидкого $\operatorname{Pr}$ и $\mathrm{Nd}$, подробно описана в работе [28]. Эти результаты были представлены ранее в работах $[24,25]$.

Оптические свойства твердых металлических пленок измерялись на воздухе при аналогичном угле падения $\varphi=82^{\circ}$ с использованием геометрии Кречмана [29], схема измерений также представлена в работе [28]. Поликристаллические пленки наносились на нижнюю грань 45-градусной равнобедренной призмы путем вакуумного термического испарения металла с вольфрамового испарителя. Полученные пленки имели толщину от 450 до $650 \mathrm{~nm}$. Толщина пленок измерялась микровзвешиванием подложки до и после напыления на весах Shimadzu AUW120D. C внешней стороны они сразу же в условиях вакуума покрывались пассивирующим слоем 
алюминия с другого испарителя, позволяющим пленке редкоземельного металла оставаться неокисленной на воздухе продолжительное время (до нескольких недель). В отличие от пленки церия [30] защита поверхности пленок $\operatorname{Pr}$ и $\mathrm{Nd}$ не была необходимостью, а представляла собой лишь дополнительную меру. Согласно рентгенофазовому анализу, полученные в данных условиях поликристаллические пленки празеодима и неодима имели $\alpha$-решетку (гексагональную плотно упакованную, ГПУ).

\section{Результаты и их обсуждение}

\section{Оптические свойства жсидкого и твердого $\mathrm{Pr}$}

Нейтральный атом празеодима имеет электронную конфигурацию внешних уровней $4 f^{3} 6 s^{2}$, трехвалентный ион - $4 f^{2} 5 s^{2} 5 p^{6}$.

Празеодим в отличие от лантана и церия имеет всего две модификации кристаллической структуры: $\alpha$ (ГПУ) и $\beta$ (объемно центрированная кубическая, ОЦК). При окислении празеодим образует оксид $\operatorname{Pr}_{6} \mathrm{O}_{11}$ [31].

В табл. 1 приведены зависимости оптических постоянные жидкого празеодима, измеренные при температуре $1273 \mathrm{~K}\left(T_{\text {melt }}=1208 \mathrm{~K}\right)[24]$, и пленки - при комнатной температуре. В данном диапазоне длин волн показатель преломления жидкого празеодима возрастает от 1.61 до 5.24 , а коэффициент поглощения соответственно от 3.10 до 6.63 , оптические постоянные пленки изменяются: $n-$ от 1.13 до $3.51, k$ - от 1.78 до 7.33 .

На рис. 1 представлены зависимости световой проводимости $\sigma$ от энергии фотонов жидкого празеодима и поликристаллической пленки. На зависимости $\sigma$ жидкого празеодима присутствуют два ярко выраженных максимума при энергиях 1.24 и $1.90 \mathrm{eV}$, а для пленки при 1.24 и $1.77 \mathrm{eV}$.

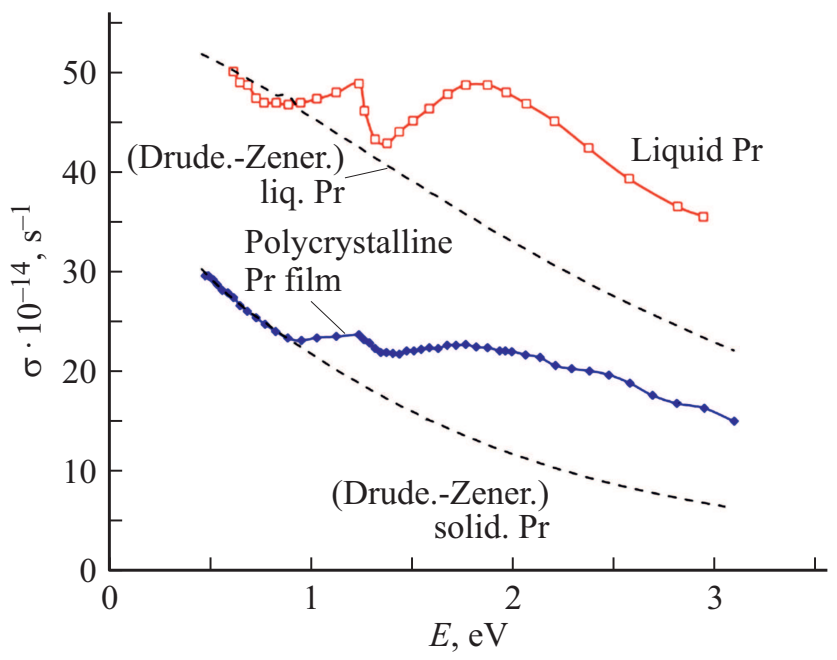

Рис. 1. Зависимости световой проводимости $\sigma$ жидкого празеодима и поликристаллической пленки от энергии фотонов $E$. Штриховыми линиями обозначен общий вклад от $s$ и $d$-электронов в световую проводимость, рассчитанный из формул Друде-Зинера.

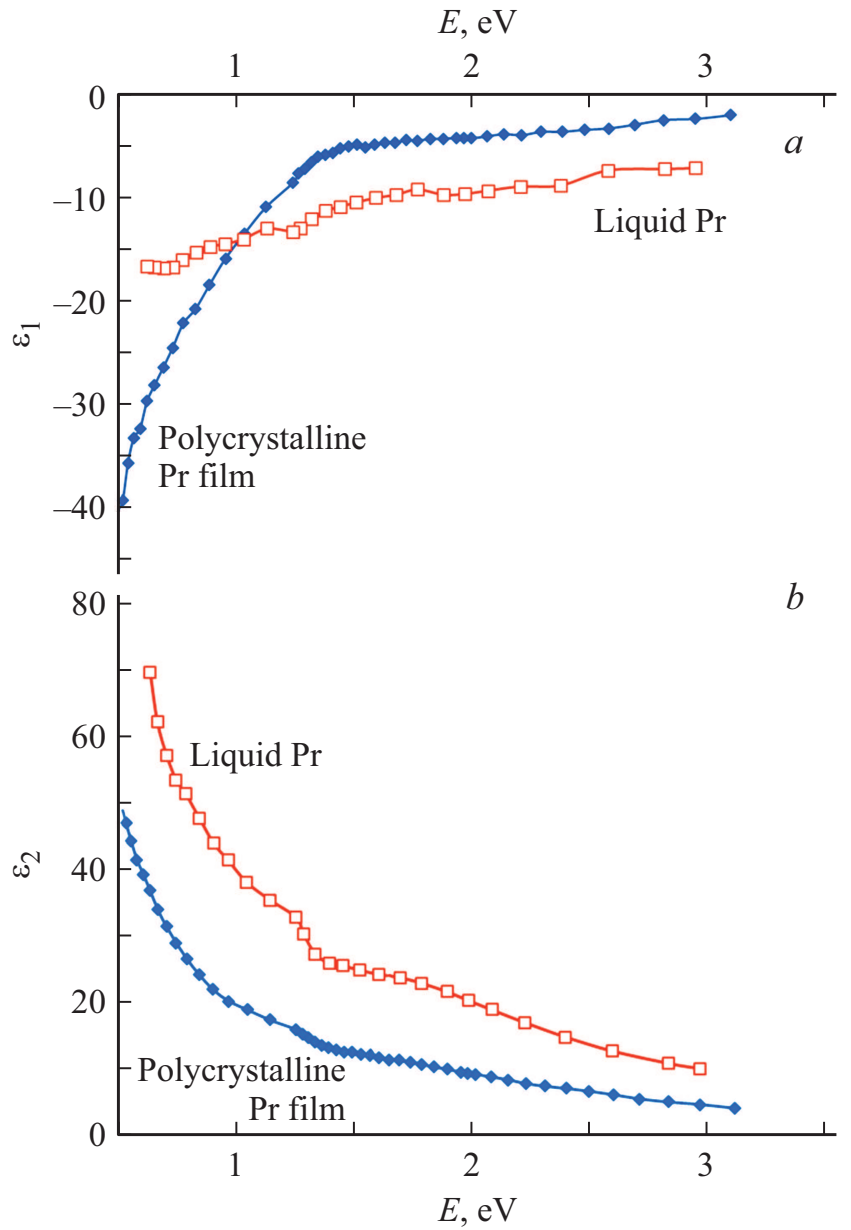

Pис. 2. (a) Дисперсионные зависимости действительной $\varepsilon_{1}(a)$ и мнимой $\varepsilon_{2}(b)$ частей диэлектрической проницаемости жидкого празеодима и пленки.

Оптические свойства жидкого и твердого празеодима в использованном интервале энергий фотонов $0.62-2.95 \mathrm{eV}$ в основном определяются межполосными переходами, как можно увидеть из рис. 1-3, на которых представлены дисперсионные зависимости $\sigma, \varepsilon_{1}$, $\varepsilon_{2}, R, \operatorname{Im}(\varepsilon)^{-1}$. Указанные дисперсионные зависимости являются немонотонными, имеют ряд особенностей. Отражательная способность $R$ изменяется от 60 до 75\% для жидкого празеодима и от 42 до 82\% для пленки, при энергиях фотонов $\hbar \omega<0.9 \mathrm{eV}, R$ монотонно возрастает при уменьшении энергии фотонов (рис. 3, $a$ ).

Функции характеристических потерь энергии электронов $\operatorname{Im}(\varepsilon)^{-1}$ при возрастании энергии фотонов увеличиваются (рис. 3, $b$ ).

В работах $[14,15]$ показано, что $4 f$-состояния празеодима расположены на $3.4 \mathrm{eV}$ ниже уровня Ферми. На основании этих данных можно предположить, что в оптическом поглощении жидкого и твердого празеодима присутствует вклад от переходов с $4 f$-состояний в полосы проводимости вблизи этих энергий. Действительно, авторы работы [11] обнаружили в оптическом спектре 
Таблица 1. Зависимости оптических постоянных жидкого празеодима и поликристаллической пленки от длины волны

\begin{tabular}{|c|c|c|c|c|c|c|c|c|c|}
\hline $\begin{array}{c}\lambda, \\
\mu \mathrm{m}\end{array}$ & $\begin{array}{c}n, \\
\text { пленка }\end{array}$ & $\begin{array}{c}k, \\
\text { пленка }\end{array}$ & $\begin{array}{c}n, \\
\text { жидкость } \\
{[24]}\end{array}$ & $\begin{array}{c}k, \\
\text { жидкость } \\
{[24]}\end{array}$ & $\begin{array}{c}\lambda, \\
\mu \mathrm{m}\end{array}$ & $\begin{array}{c}n, \\
\text { пленка }\end{array}$ & $\begin{array}{c}k, \\
\text { пленка }\end{array}$ & $\begin{array}{c}n, \\
\text { жидкость } \\
{[24]}\end{array}$ & $\begin{array}{c}k, \\
\text { жидкость } \\
{[24]}\end{array}$ \\
\hline 0.4 & 1.13 & 1.78 & & & 0.86 & 2.05 & 3.05 & 2.9 & 4.38 \\
\hline 0.42 & 1.20 & 1.92 & 1.61 & 3.10 & 0.88 & 2.06 & 3.12 & - & - \\
\hline 0.44 & 1.25 & 1.98 & 1.7 & 3.16 & 0.9 & 2.08 & 3.17 & 2.91 & 4.43 \\
\hline 0.46 & 1.28 & 2.11 & - & - & 0.92 & 2.10 & 3.21 & - & - \\
\hline 0.48 & 1.35 & 2.24 & 1.91 & 3.3 & 0.94 & 2.12 & 3.31 & 2.98 & 4.56 \\
\hline 0.5 & 1.43 & 2.31 & - & - & 0.96 & 2.15 & 3.42 & - & - \\
\hline 0.52 & 1.47 & 2.38 & 2.05 & 3.59 & 0.98 & 2.17 & 3.50 & 3.16 & 4.78 \\
\hline 0.54 & 1.52 & 2.41 & - & - & 1 & 2.18 & 3.63 & 3.32 & 4.92 \\
\hline 0.56 & 1.55 & 2.49 & 2.28 & 3.7 & 1.1 & 2.19 & 3.95 & 3.51 & 5.02 \\
\hline 0.58 & 1.64 & 2.53 & - & - & 1.2 & 2.20 & 4.27 & 3.64 & 5.21 \\
\hline 0.6 & 1.68 & 2.60 & 2.42 & 3.88 & 1.3 & 2.21 & 4.55 & 3.83 & 5.39 \\
\hline 0.62 & 1.72 & 2.66 & - & - & 1.4 & 2.26 & 4.84 & 3.97 & 5.51 \\
\hline 0.63 & 1.74 & 2.67 & 2.53 & 3.99 & 1.5 & 2.36 & 5.12 & 4.2 & 5.73 \\
\hline 0.64 & 1.76 & 2.68 & - & - & 1.6 & 2.49 & 5.32 & 4.36 & 5.88 \\
\hline 0.66 & 1.81 & 2.73 & 2.64 & 4.07 & 1.7 & 2.59 & 5.58 & 4.43 & 6.02 \\
\hline 0.68 & 1.85 & 2.76 & - & - & 1.8 & 2.70 & 5.80 & 4.73 & 6.25 \\
\hline 0.7 & 1.89 & 2.81 & 2.78 & 4.10 & 1.9 & 2.82 & 6.00 & 4.92 & 6.31 \\
\hline 0.72 & 1.92 & 2.83 & - & - & 2 & 2.96 & 6.20 & 5.24 & 6.63 \\
\hline 0.74 & 1.94 & 2.88 & 2.82 & 4.19 & 2.1 & 3.04 & 6.44 & - & - \\
\hline 0.76 & 1.96 & 2.89 & - & - & 2.2 & 3.15 & 6.57 & - & - \\
\hline 0.78 & 1.98 & 2.95 & 2.85 & 4.23 & 2.3 & 3.25 & 6.80 & - & - \\
\hline 0.8 & 1.99 & 3.00 & - & - & 2.4 & 3.31 & 7.08 & - & - \\
\hline 0.82 & 2.03 & 2.98 & 2.87 & 4.31 & 2.5 & 3.43 & 7.21 & - & - \\
\hline 0.84 & 2.05 & 3.02 & - & - & 2.6 & 3.51 & 7.33 & - & - \\
\hline
\end{tabular}

празеодима пик световой проводимости при энергии фотонов $3.05 \mathrm{eV}$. К сожалению, величины этих энергий находятся за пределами использованного в настоящей работе спектрального диапазона.

На рис. 4 приведены результаты расчета по теории почти свободных электронов в модели гибридизированной $s-d$-полосы $(N-$ концентрация электронов проводимости, $\gamma$ - частота релаксации жидкого празеодима, $\sigma(0)$ - предельная световая проводимость для спектрального интервала $\hbar \omega<1.24 \mathrm{eV})$. В предположении, что в ИК области $(\lambda=2 \mu \mathrm{m})$ вклад в световую проводимость от межполосных переходов незначителен по сравнению с вкладом от внутриполосного ускорения электронов, рассчитывалась эффективная концентрация носителей $l$, приходящаяся на один атом:

$$
l=\frac{N_{e} A}{d N_{\mathrm{A}}},
$$

где $N_{e}$ - концентрация электронов проводимости, $A-$ атомная масса, $d$ - плотность жидкого празеодима, $N_{\mathrm{A}}=6.02 \cdot 10^{23} \mathrm{~mol}^{-1}$ - число Авогадро.

Величина $l$ оказалась равной $2.94 \mathrm{el} / \mathrm{at}$. Зная концентрацию носителей заряда для жидкого празеодима, рассчитывалась плазменная частота. Для объемных плазмонов $\omega_{\mathrm{pv}}=16.22 \cdot 10^{15} \mathrm{~s}^{-1}$, что соответствует энергии объемных плазмонов $\hbar \omega_{\mathrm{pv}}=10.68 \mathrm{eV}$. Для поверхностных плазмонов, согласно расчету, $\hbar \omega_{\mathrm{ps}}=7.55 \mathrm{eV}$. Сведения по экспериментальному измерению энергий объемных и поверхностных плазмонов празеодима в литературе отсутствуют.

Проводимость на постоянном токе $\sigma(0)$, рассчитанная по найденным значениям $N$ и $\gamma$, представлена на рис. 4, $c$. Предельное значение $\sigma(0)=5.6 \cdot 10^{5} \Omega^{-1} \mathrm{~m}^{-1}$ для жидкого празеодима меньше удельной статической проводимости $\sigma_{\text {stat }}=7.14 \cdot 10^{5} \Omega^{-1} \mathrm{~m}^{-1}$, взятой из работы [32].

\section{Оптические свойства жсидкого и твердого $\mathrm{Nd}$}

Атом неодима имеет электронную конфигурацию внешних уровней $4 f^{4} 6 s^{2}$, а трехвалентный ион $4 f^{3} 5 s^{2} 5 p^{6}$.

Неодим имеет, как и празеодим, две модификации кристаллической структуры $\alpha$ (ГПУ), $\beta$ (ОЦК) [31]. Как правило, неодим при окислении образует оксид $\mathrm{Nd}_{2} \mathrm{O}_{3}$.

Результаты измерений оптических постоянных жидкого неодима при температуре $1353 \mathrm{~K}\left(T_{\text {melt }}=1297 \mathrm{~K}[25]\right)$ и поликристаллической пленки при комнатной температуре приведены в табл. 2. Из табл. 2 видно, что в указанном интервале спектра показатель преломления жидкого неодима возрастает от 1.65 до 5.1, а коэффициент поглощения - от 2.98 до 6.24, $n$ пленки возрастает от 1.09 до $3.86, k$ - от 2.01 до 5.95. Подобные закономерности проявлялись на жидких и твердых церии и празеодиме.

Дисперсионные зависимости световой проводимости $\sigma$, отражательной способности $R$, мнимой и действи- 
Таблица 2. Зависимости оптических постоянных жидкого и твердого неодима от длины волны

\begin{tabular}{|c|c|c|c|c|c|c|c|c|c|}
\hline $\begin{array}{c}\lambda, \\
\mu \mathrm{m}\end{array}$ & $\begin{array}{c}n, \\
\text { пленка }\end{array}$ & $\begin{array}{c}k, \\
\text { пленка }\end{array}$ & $\begin{array}{c}n, \\
\text { жидкость } \\
{[26]}\end{array}$ & $\begin{array}{c}k, \\
\text { жидкость } \\
{[26]}\end{array}$ & $\begin{array}{c}\lambda, \\
\mu \mathrm{m}\end{array}$ & $\begin{array}{c}n, \\
\text { пленка }\end{array}$ & $\begin{array}{c}k, \\
\text { пленка }\end{array}$ & $\begin{array}{c}n, \\
\text { жидкость } \\
{[26]}\end{array}$ & $\begin{array}{c}k, \\
\text { жидкость } \\
{[26]}\end{array}$ \\
\hline 0.4 & 1.09 & 2.01 & - & - & 0.86 & 2.22 & 3.43 & - & - \\
\hline 0.42 & 1.19 & 2.11 & - & - & 0.88 & 2.23 & 3.46 & 3.48 & 4.42 \\
\hline 0.44 & 1.23 & 2.22 & 1.65 & 2.98 & 0.92 & - & - & 3.59 & 4.47 \\
\hline 0.46 & 1.31 & 2.35 & - & - & 0.94 & 2.17 & 3.70 & - & - \\
\hline 0.48 & 1.36 & 2.45 & 1.91 & 3.40 & 0.96 & 2.15 & 3.78 & 3.69 & 4.69 \\
\hline 0.5 & 1.41 & 2.52 & - & - & 0.98 & 2.13 & 3.82 & - & - \\
\hline 0.52 & 1.43 & 2.60 & 2.19 & 3.59 & 1 & 2.18 & 3.84 & 3.77 & 4.92 \\
\hline 0.54 & 1.48 & 2.67 & - & - & 1.1 & 2.31 & 4.09 & 3.78 & 5.13 \\
\hline 0.56 & 1.53 & 2.76 & 2.36 & 3.74 & 1.2 & 2.41 & 4.36 & 3.81 & 5.36 \\
\hline 0.58 & 1.59 & 2.81 & - & - & 1.3 & 2.55 & 4.59 & 3.88 & 5.41 \\
\hline 0.6 & 1.64 & 2.85 & 2.55 & 3.88 & 1.4 & 2.72 & 4.78 & 3.97 & 5.45 \\
\hline 0.62 & 1.68 & 2.89 & - & - & 1.5 & 2.81 & 5.04 & 4.07 & 5.49 \\
\hline 0.63 & 1.73 & 2.93 & - & - & 1.6 & 2.98 & 5.19 & 4.17 & 5.53 \\
\hline 0.64 & 1.77 & 2.98 & 2.8 & 3.96 & 1.7 & 3.05 & 5.40 & 4.39 & 5.58 \\
\hline 0.66 & 1.87 & 3.03 & - & - & 1.8 & 3.21 & 5.51 & 4.57 & 5.84 \\
\hline 0.68 & 1.92 & 3.09 & 2.89 & 4.01 & 1.9 & 3.21 & 5.85 & 4.78 & 6.02 \\
\hline 0.7 & 1.96 & 3.12 & - & - & 2 & 3.37 & 5.95 & 5.1 & 6.24 \\
\hline 0.72 & 2.03 & 3.15 & 2.95 & 4.11 & 2.1 & 3.37 & 6.25 & - & - \\
\hline 0.74 & 2.04 & 3.19 & - & - & 2.2 & 3.46 & 6.40 & - & - \\
\hline 0.76 & 2.09 & 3.21 & 3.07 & 4.23 & 2.3 & 3.52 & 6.58 & - & - \\
\hline 0.78 & 2.14 & 3.23 & - & - & 2.4 & 3.67 & 6.68 & - & - \\
\hline 0.8 & 2.16 & 3.29 & 3.19 & 4.29 & 2.5 & 3.79 & 6.76 & - & - \\
\hline 0.82 & 2.20 & 3.32 & - & - & 2.6 & 3.86 & 6.95 & - & - \\
\hline 0.84 & 2.21 & 3.34 & 3.32 & 4.35 & & & & & \\
\hline
\end{tabular}

тельной частей диэлектрической проницаемости $\varepsilon_{2}$ и $\varepsilon_{1}$, функций характеристических потерь энергии $\operatorname{Im}(\varepsilon)^{-1}$ жидкого и твердого неодима имеют особенности, вызванные межполосными переходами (рис. 5-7). Из рис. 5 видно, что дисперсионная зависимость световой проводимости $\sigma$ жидкого неодима имеет два максимума при энергиях фотонов 1.24 и $1.95 \mathrm{eV}$, а на $\sigma(\omega)$ тонкой пленки наблюдается широкая полоса поглощения в области $1.27-2.00 \mathrm{eV}$. Согласно работе [15], $4 f$-уровни неодима расположены на энергии $\sim 4.8 \mathrm{eV}$ ниже $E_{\mathrm{F}}$. Такая энергия находится вне спектрального диапазона, используемого в настоящей работе.

Отражательная способность $R$ жидкого неодима в данном спектральном диапазоне изменяется от 58 до $73 \%$, а пленки - от 47 до 80\% (рис. 7, $a$ ).

Функции характеристических потерь энергии электронов при переходе от ИК диапазона к ультрафиолетовому возрастают (рис. 7, $b$ ).

На рис. 8 для спектрального интервала $\hbar \omega<1.24 \mathrm{eV}$ приведены результаты расчета концентрации электронов проводимости $N$, частоты релаксации для жидкого неодима $\gamma$ и предельной световой проводимости $\sigma(0)$ по теории почти свободных электронов в модели гибридизированной $s-d$-полосы. Для $\lambda=2 \mu \mathrm{m}$ рассчитывалась эффективная концентрация носителей, приходящаяся на один атом. Ее величина оказалась равной 2.95. Зная эффективную концентрацию носителей для жидкого неоди- ма, рассчитывалась плазменная частота. Для объемных плазмонов $\omega_{\mathrm{pv}}=16.53 \cdot 10^{15} \mathrm{~s}^{-1}$, что соответствует их энергии $\hbar \omega_{\mathrm{pv}}=10.88 \mathrm{eV}$. Для поверхностных плазмонов энергия равна $\hbar \omega_{\mathrm{ps}}=7.69 \mathrm{eV}$. Сведения по экспериментальному измерению энергий плазмонов неодима в литературе отсутствуют.

Проводимость на постоянном токе $\sigma(0)$ представлена на рис. 8. Предельное значение $\sigma(0)=5.05 \cdot 10^{5} \Omega^{-1} \cdot \mathrm{m}^{-1}$ при $\lambda=2 \mu \mathrm{m}$ для жидкого неодима меньше удельной статической проводимости $\sigma_{\text {stat }}=6.67 \cdot 10^{5} \Omega^{-1} \cdot \mathrm{m}^{-1}$, взятой из работы [32].

Модель однополосной проводимости почти свободных электронов применима к электронам проводимости непереходных металлов. Для описания оптических свойств переходных металлов автором [33,34] предложена модель двухполосной проводимости, согласно которой проводимость переходных металлов обусловлена вкладом медленно релаксирующих ( $s$-подобных) и быстро релаксирующих ( $d$-подобных) электронов, отличающихся плазменными $\omega_{p s}^{2}=4 \pi N_{s} e^{2} / m, \omega_{p d}^{2}=4 \pi N_{d} e^{2} / m$ $\left(N_{s}, N_{d}, m, e-\right.$ концентрации, масса и заряд $s$ - и $d$-электронов соответственно) и релаксационными частотами, $\gamma_{s} \ll \gamma_{d}$. Нами указанная модель использована для расчета электронных характеристик церия и иттербия в конденсированном состоянии [28,30].

Модель двухполосной проводимости позволяет определить вклады от $s$ - и $d$-электронов в предельную 

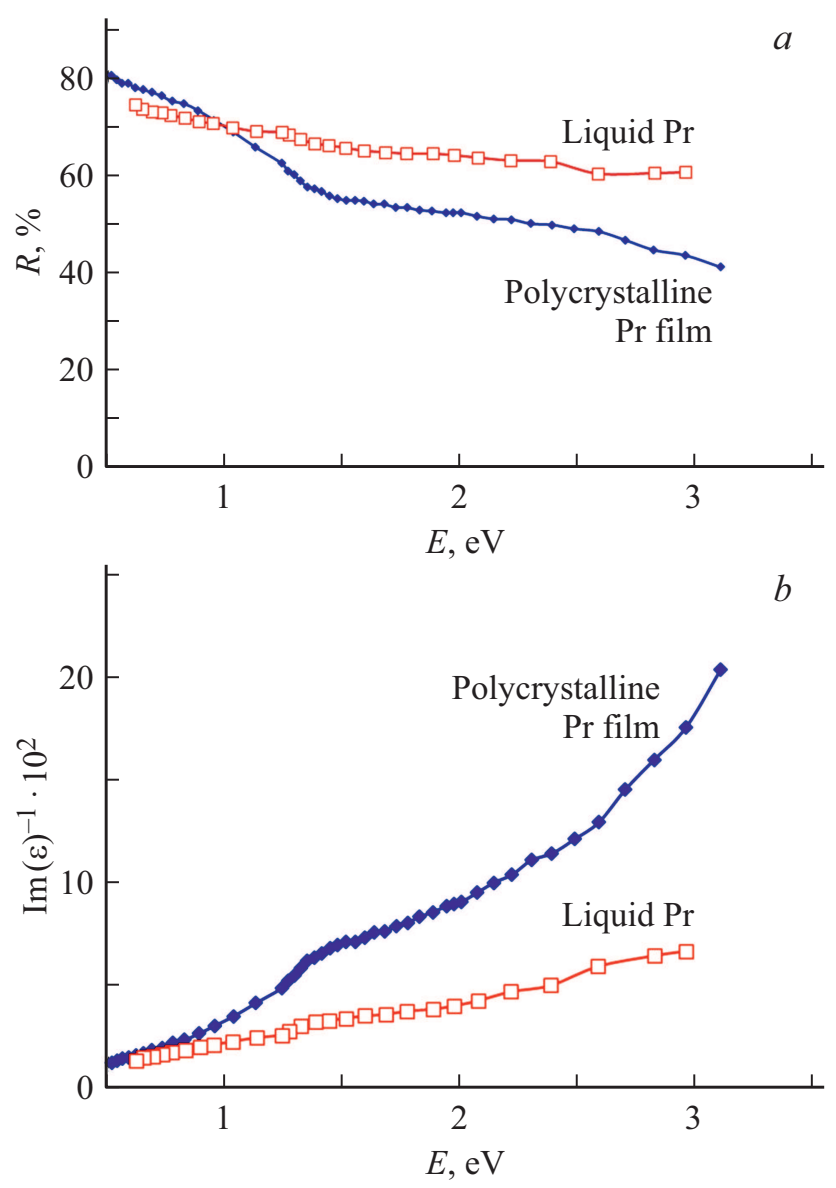

Pис. 3. (a) Дисперсионные зависимости отражательной способности $R$ жидкого празеодима и пленки. $(b)$ Функции характеристических потерь энергии электронов жидкого празеодима и пленки.

световую проводимость:

$$
\sigma_{0 s}=\frac{\omega_{p s}^{2}}{4 \pi \gamma_{s}} ; \quad \sigma_{0 d}=\frac{\omega_{p d}^{2}}{4 \pi \gamma_{d}} ; \quad \sigma_{0}=\sigma_{0 s}+\sigma_{0 d} .
$$

Таким образом определяются все электронные характеристики: $\quad \gamma_{s}, \gamma_{d}, \omega_{p s}^{2}, \omega_{p d}^{2}, N_{s}, N_{d}, P, \sigma_{0 s}, \sigma_{0 d}$, $\sigma_{0}=\sigma_{0 s}+\sigma_{0 d}$ (предельная световая проводимость). В табл. 3 и 4 приводятся электронные характеристики, рассчитанные для $\operatorname{Pr}$ и $\mathrm{Nd}$ в жидком и твердом тонкопленочном состояниях.

При плавлении празеодима концентрация носителей заряда существенно увеличивается (из расчета по модели двухполосной проводимости), а предельная световая проводимость уменьшается в два раза, как и статическая проводимость. В табл. 4 также приведены характеристики электронов проводимости жидкого празеодима и неодима, рассчитанные по модели однополосной проводимости. Следует отметить, что для жидких празеодима и неодима расчеты характеристик электронов проводимости по указанным моделям дают близкие результаты по их концентрации $N_{e}$, эффективной концентрации $l$, предельной световой проводимости $\sigma(0)$.
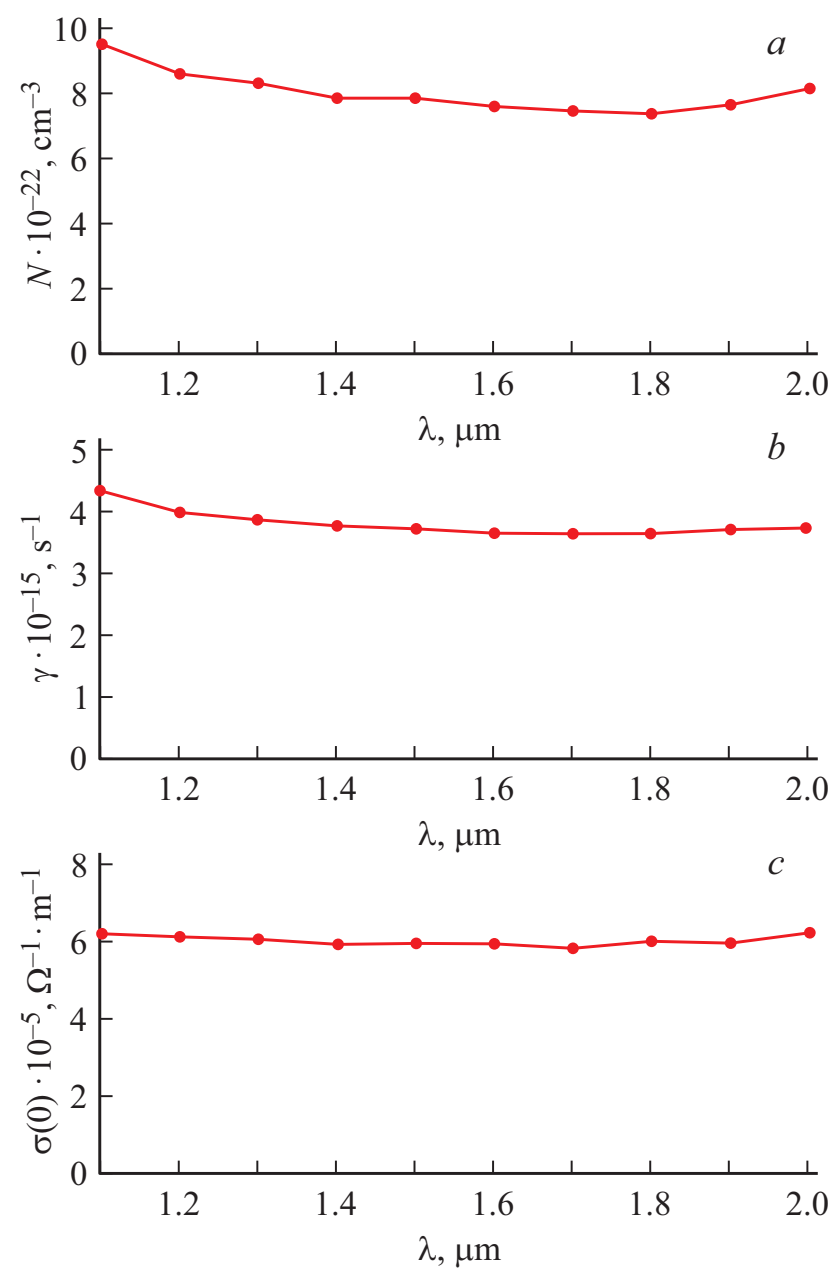

Рис. 4. Дисперсионные зависимости концентрации носителей заряда $N(a)$, частоты релаксации $\gamma(b)$ и проводимости $\sigma(0)(c)$, жидкого празеодима, рассчитанные по модели однополосной проводимости.

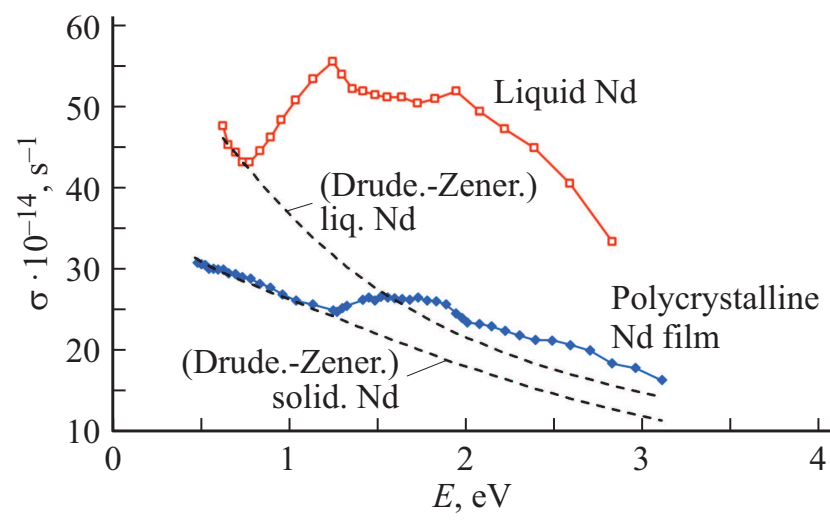

Рис. 5. Зависимости световой проводимости $\sigma$ жидкого и твердого неодима от энергии фотонов $E$. Штриховыми линиями обозначен общий вклад от $s$ - и $d$-электронов в световую проводимость, рассчитанный по формулам Друде-Зинера.

При плавлении неодима концентрация носителей заряда также увеличивается, а предельная световая про- 
Таблица 3. Характеристики электронов проводимости для $\operatorname{Pr}$ и $\mathrm{Nd}$ в жидком и твердом состояниях

\begin{tabular}{c|l|c|l|c|c|c|c|c}
\hline Me & $\begin{array}{c}\gamma_{s} \cdot 10^{-15}, \\
\mathrm{~s}^{-1}\end{array}$ & $\begin{array}{c}\gamma_{d} \cdot 10^{-15}, \\
\mathrm{~s}^{-1}\end{array}$ & $\begin{array}{l}\omega_{p s}^{2} \cdot 10^{-30}, \\
\mathrm{~s}^{-2}\end{array}$ & $\begin{array}{c}\omega_{p d}^{2} \cdot 10^{-30}, \\
\mathrm{~s}^{-2}\end{array}$ & $P$ & $\begin{array}{c}N_{s} \cdot 10^{-22}, \\
\mathrm{~cm}^{-3}\end{array}$ & $\begin{array}{c}N_{d} \cdot 10^{-22}, \\
\mathrm{~cm}^{-3}\end{array}$ & $\begin{array}{c}\left(N_{s}+N_{d}\right) \cdot 10^{-22}, \\
\mathrm{~cm}^{-3}\end{array}$ \\
\hline $\mathrm{Pr}_{\text {solid }}$ & 0.136 & 2.49 & 16.52 & 90.58 & 2 & 0.52 & 2.84 & \\
$\mathrm{Pr}_{\text {liquid }}$ & 1.39 & 4.11 & 10.97 & 258.79 & 2 & 0.34 & 8.13 & 3.36 \\
$\mathrm{Nd}_{\text {solid }}$ & 0.11 & 3.68 & 13.31 & 139.82 & 1 & 0.42 & 4.39 & 8.47 \\
$\mathrm{Nd}_{\text {liquid }}$ & 1.65 & 6.85 & 88.2 & 123.6 & 13 & 2.77 & 3.88 & 6.81 \\
\end{tabular}

Таблица 4. Проводимости $\operatorname{Pr}$ и $\mathrm{Nd}$ в жидком и твердом состояниях

\begin{tabular}{|c|c|c|c|c|c|c|}
\hline Металл & $\begin{array}{c}l, \\
\text { el./at. }\end{array}$ & $\begin{array}{l}\sigma_{0 s} \cdot 10^{-5} \\
\Omega^{-1} \cdot \mathrm{m}^{-1}\end{array}$ & $\begin{array}{l}\sigma_{0 d} \cdot 10^{-5} \\
\Omega^{-1} \cdot \mathrm{m}^{-1}\end{array}$ & $\begin{array}{c}\sigma_{0} \cdot 10^{-5} \\
\Omega^{-1} \cdot \mathrm{m}^{-1}\end{array}$ & $\begin{array}{l}\sigma_{\mathrm{cr}} \cdot 10^{-5} \\
\Omega^{-1} \cdot \mathrm{m}^{-1}\end{array}$ & $\begin{array}{c}N_{e} \cdot 10^{-22} \\
\mathrm{~cm}^{-3}\end{array}$ \\
\hline $\operatorname{Pr}_{\text {solid }}$ & 1.16 & 10.77 & 3.21 & 13.98 & 14.29 & 3.36 \\
\hline $\operatorname{Pr}_{\text {liquid }}$ & 3.08 & 0.7 & 5.58 & 6.28 & 7.14 & 8.47 \\
\hline $\begin{array}{c}\operatorname{Pr}_{\text {liquid }} \\
\text { (расчет по } \\
\text { однополосной } \\
\text { модели [33]) }\end{array}$ & 2.94 & - & - & 5.6 & 7.14 & 8.18 \\
\hline $\mathrm{Nd}_{\text {solid }}$ & 1.65 & 11.07 & 3.36 & 14.43 & 15.55 & 4.81 \\
\hline $\mathrm{Nd}_{\text {liquid }}$ & 2.43 & 4.72 & 1.6 & 6.32 & 6.67 & 6.65 \\
\hline $\begin{array}{c}\mathrm{Nd}_{\text {liquid }} \\
\text { (расчет по } \\
\text { однополосной } \\
\text { модели [33]) }\end{array}$ & 2.95 & - & - & 5.05 & 6.67 & 8.49 \\
\hline
\end{tabular}

водимость уменьшается в 2.3 раза, как и статическая проводимость.

Оптические свойства, определяемые особенностями энергетического спектра электронов, весьма чувствительны к фазовым и магнитным превращениям в металлах и сплавах, так как вследствие изменения параметров решетки, характера ее симметрии и магнитного упорядочения изменяется энергетическое состояние валентных электронов.

Исчезновение кристаллической решетки при плавлении металлов приводит к повышению частоты релаксации электронов, уменьшению статической проводимости и возрастанию концентрации носителей заряда [33]. Известно, что частота релаксации электронов проводимости состоит из трех слагаемых:

$$
\gamma=\gamma_{e p}+\gamma_{e e}+\gamma_{e d}
$$

где $\gamma_{e p}$ - частота релаксации электронов, связанная с процессами взаимодействия электронов проводимости с фононами, $\gamma_{e e}$ - частота межэлектронных взаимодействий, $\gamma_{e d}-$ частота взаимодействия электронов с примесями. Обычно величина $\gamma_{e e} \ll \gamma$, и ее можно наблюдать лишь при гелиевых температурах. В чистых металлах величина $\gamma_{e d}$ также мала. Таким образом, плавление металла приводит к повышению частоты релаксации взаимодействия электронов с фононами. В табл. 3 видно, что при плавлении празеодима и неодима возрастают частоты релаксации $\gamma_{s}$ и $\gamma_{d}$ для $s-$ и $d$-электронов.

Расчет общих вкладов от $s-\boldsymbol{u} d$-электронов в световую проводимость $\sigma(\omega)$

В случае переходных металлов и их сплавов световая проводимость $\sigma(\omega)$ и действительная часть диэлектрической проницаемости $\varepsilon_{1}(\omega)$ определяются вкладами межзонных переходов $\sigma_{m z}$ и внутризонных, обусловленных $s$ - и $d$-электронами проводимости $\sigma_{s}(\omega)$ и $\sigma_{d}(\omega)$ :

$$
\sigma(\omega)=\sigma_{m z}+\sigma_{s}(\omega)+\sigma_{d}(\omega)
$$

Вклады в световую проводимость $\sigma_{s}(\omega)$ и $\sigma_{d}(\omega)$ рассчитываются по формулам Друде-Зинера [33]:

$$
\begin{aligned}
& \sigma_{s}(\omega)=\frac{\omega_{p s}^{2} \gamma_{s}}{\left(\omega^{2}+\gamma_{s}^{2}\right) 4 \pi}, \\
& \sigma_{d}(\omega)=\frac{\omega_{p d}^{2} \gamma_{d}}{\left(\omega^{2}+\gamma_{d}^{2}\right) 4 \pi} .
\end{aligned}
$$

Используя характеристики электронов проводимости поликристаллических и жидких $\operatorname{Pr}$ и $\mathrm{Nd}, \gamma_{s}, \gamma_{d}, \omega_{p s}^{2}, \omega_{p d}^{2}$, представленные в табл. 3, по уравнениям (5) и (6) 

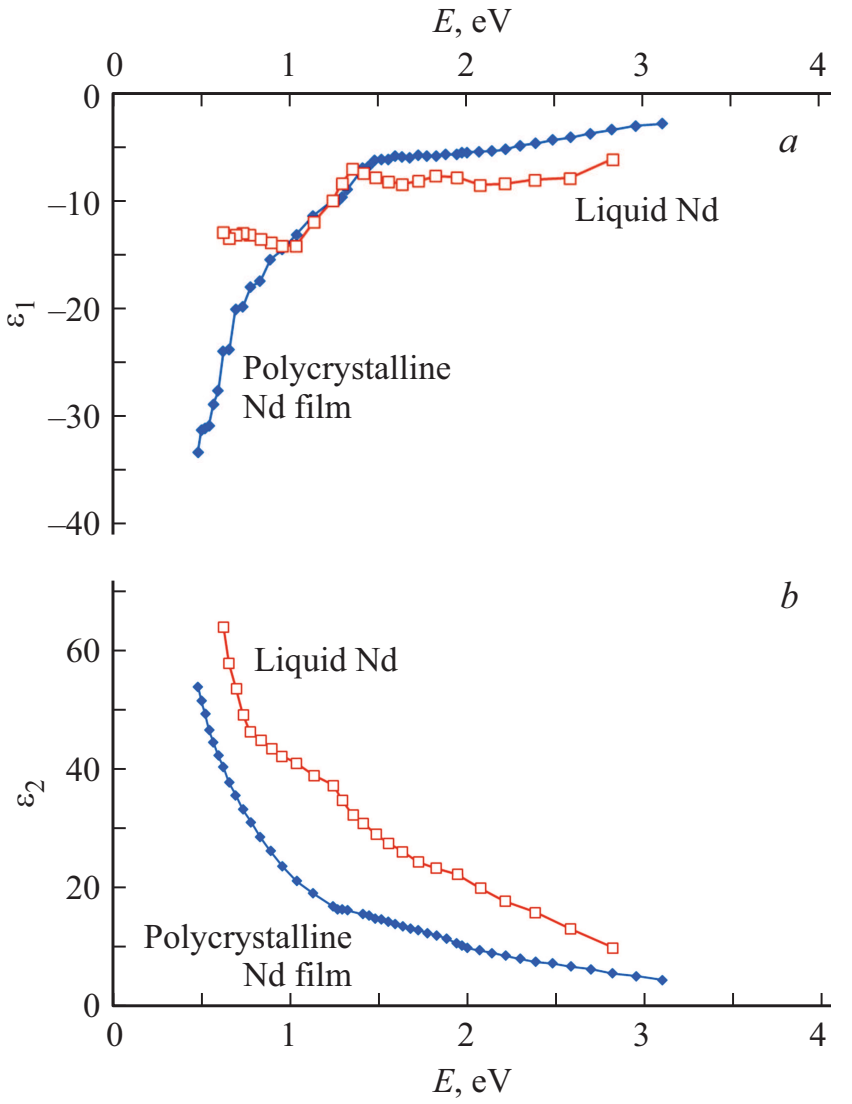

Рис. 6. Дисперсионные зависимости действительной $\varepsilon_{1}(a)$ и мнимой $\varepsilon_{2}(b)$ частей диэлектрической проницаемости жидкого неодима и пленки.

рассчитаны вклады в световую проводимость в использованном спектральном диапазоне. На рис. 1 и 5 штриховыми линиями нанесены эти зависимости для жидких и твердых $\mathrm{Pr}$ и $\mathrm{Nd}$. Из этих рисунков видно, что при энергии $\hbar \omega<0.59 \mathrm{eV}(\lambda>2 \mu \mathrm{m})$ световая проводимость определяется внутризонными переходами.

\section{Заключение}

Эллипсометрическим методом Битти исследованы оптические свойства неокисленной поверхности поликристаллических пленок празеодима и неодима в спектральном диапазоне $0.4-2.6 \mu \mathrm{m}$. На основе оптических постоянных пленок рассчитаны дисперсионные зависимости световой проводимости, действительной и мнимой частей диэлектрической проницаемости, отражательной способности и функций характеристических потерь энергии электронов. Приводятся спектры оптических свойств как пленок, так и жидких металлов, исследованных ранее.

Установлено, что оптические свойства жидких и твердых празеодима и неодима в указанном спектральном интервале определяются в основном межполосными переходами. На дисперсионных зависимостях световой проводимости этих металлов в жидком состоянии обнаружены по два максимума при энергиях 1.24 и $1.82 \mathrm{eV}$ у празеодима, 1.24 и $1.95 \mathrm{eV}-$ у неодима, 1.24 и $1.77 \mathrm{eV}$ - у твердого празеодима, а на твердом неодиме наблюдается широкая полоса поглощения в области спектра $1.27-2.0 \mathrm{eV}$. Таким образом, при плавлении данных металлов наблюдается смещение некоторых полос поглощения, вызванное разрушением кристаллической решетки.

Показано, что приближение почти свободных электронов для исследованных жидких металлов выполняется при длине волны $\lambda \geq 2 \mu \mathrm{m}$. Из теории почти свободных электронов определена эффективная концентрация электронов проводимости, приходящаяся на один атом. Для указанных жидких металлов она близка к 3, что хорошо согласуется с числом валентных электронов $(z=3)$. Предельное значение световой проводимости на постоянном токе $\sigma(0)$ меньше удельной статической проводимости $\sigma_{\text {stat }}$, что равнозначно большей величине оптической частоты релаксации. Носители заряда сильнее рассеиваются при действии на них высокочастотного поля (по сравнению со статическим).

Из расчетов по модели двухполосной проводимости на основе экспериментальных измерений в ИК области спектра следует, что при плавлении празеодима и неодима концентрации носителей заряда и частоты релаксации увеличиваются, а предельные световые про-
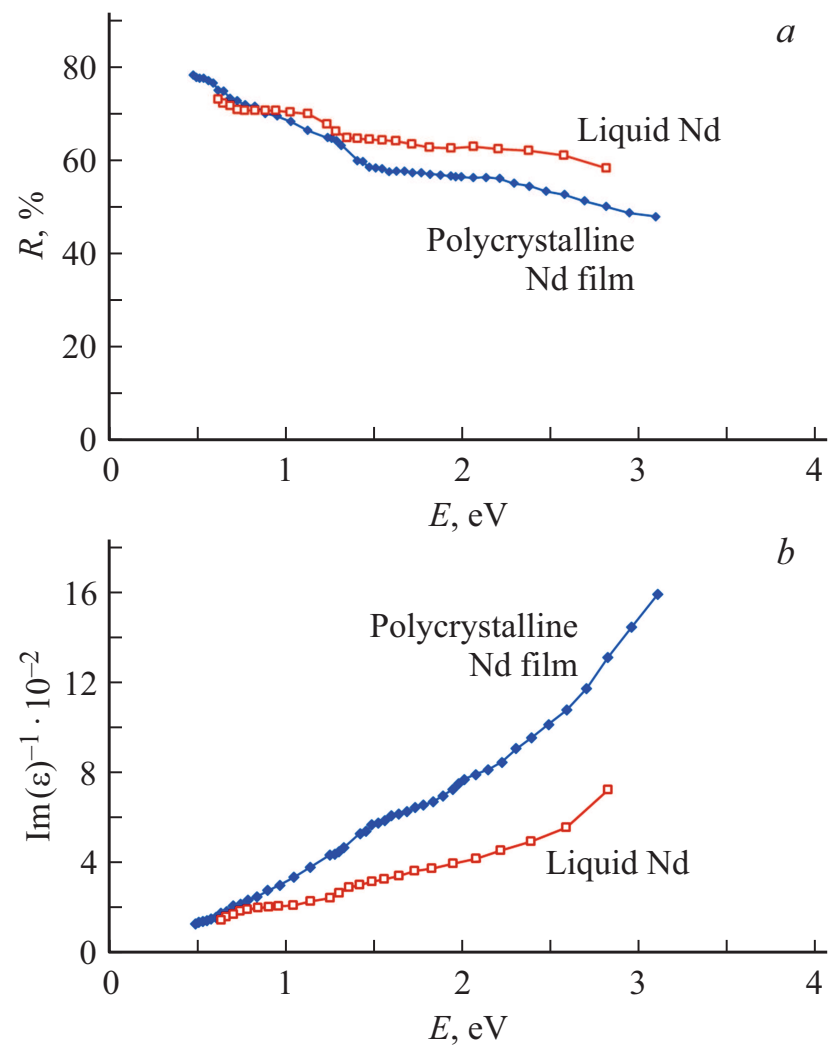

Рис. 7. Дисперсионные зависимости отражательной способности $R(a)$ и функции характеристических потерь энергии электронов $(b)$ жидкого $\mathrm{Nd}$ и пленки. 

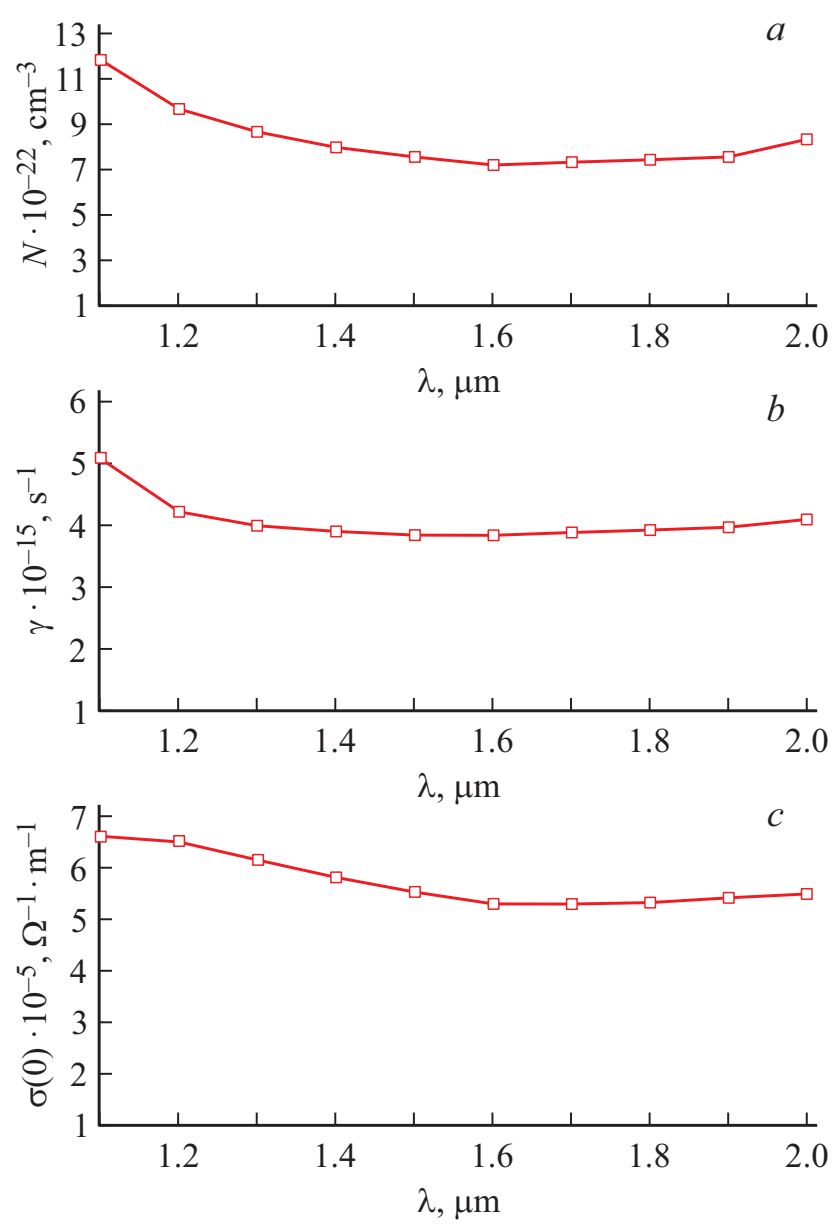

Рис. 8. Жидкий неодим. Дисперсионные зависимости концентрации носителей заряда $N(a)$, частоты релаксации $\gamma(b)$ и световой проводимости $\sigma(0)(c)$.

водимости значительно уменьшаются, как и статические проводимости. Следует отметить, что для изученных жидких металлов расчеты характеристик электронов проводимости по моделям однополосной и двухполосной проводимости дают близкие результаты по их концентрации $N_{e}$, эффективной концентрации, предельной световой проводимости $\sigma(0)$.

\section{Финансирование работы}

Работа выполнена в соответствии с гос. заданием АААА-А19-119031890028-0 (Структурное подразделение № 2).

\section{Конфликт интересов}

Авторы заявляют, что у них нет конфликта интересов.

\section{Список литературы}

[1] Рябухина М.В., Кравцов Е.А., Наумова Л.И., Проглядо В.В., Хайдуков Ю.Н., Устинов В.В // Физика ме- таллов и металловедение. 2017. Т. 118. № 2. С. 151. doi 10.7868/S0015323017020115

[2] Banerjee A., Abhilash S.R., Umapathy G.R., Kabiraj D., Ojha S., Mandal S. // Nuclear Inst. and Methods in Physics Research. A. 2018. V. 887. P. 34. doi 10.1016/j.nima.2018.01.013

[3] Banerjee A., Umapathy G.R., Abhilash S.R., Ojha S., Unnati, Kabiraj D., Mandal S. // Vacuum. 2019. V. 165. P. 68. doi 10.1016/j.vacuum.2019.04.005

[4] Mor G.K., Malhotra L.K // Thin Solid Films. 2000. V. 359. P. 28. doi 10.1016/S0040- 6090(99)00708-7

[5] Provo J.L // J. Vacuum Science \& Technology A. 2015. V. 33. N 4. P. 041507. doi $10.1116 / 1.4919929$

[6] Azofeifa D., Clark N., Vargas W. // Phys. Stat. Sol. (b). 2005. V. 242. N 10. P. 2005. doi 10.1002/pssb.200540061

[7] Tacon S.Le, Brodier A., Chicanne C., Theobald M. // Fusion Science and Technology. 2016. V. 70. N 2. P. 351. doi 10.13182/FST15-240

[8] Burnham A.K., Jameson G.T // J. Vacuum Science \& Technology A. 1987. V. 5. P. 1713. doi 10.1116/1.574515

[9] Homewood V.J., Trodahl H.J., Staines M.P. // Philosophical Magazine. Part B. 1994. V. 70. N 3. P. 499. doi 10.1080/01418639408240224

[10] Saini S.M., Singh N., Nautiyal T., Auluck S. // Solid State Commun. 2006. V. 140. P. 125. doi 10.1016/j.ssc.2006.08.016

[11] Князев Ю.В., Носков М.М // Физика металлов и металловедение. 1973. Т. 36. № 2. С. 299.

[12] Князев Ю.В., Кузьмин Ю.И., Кириллова М.М // Физика металлов и металловедение. 1995. Т. 79. № 5. С. 60.

[13] Князев Ю.В., Кузмин Ю.И., Бурханов Г.С., Чистяков О.Д., Кольчугина Н.Б // Физика металлов и металловедение. 1993. T. 76. № 6. C. 76.

[14] Baer Y., Busch G. // Phys. Rev. Lett. 1973. V. 31. N 1. P. 35. doi 10.1103/PhysRevLett.31.35

[15] Lang I.K., Baer Y., Cox P.A // J. Phys. F: Metal Phys. 1981. V. 11. N 1. P. 121. http://iopscience.iop.org/0305-4608/11/1/015

[16] Haensel R., Rabe P., Sonntag B. // Solid State Commun. 1970. V. 8. Is. 22. P. 1845. doi 10.1016/0038-1098(70)90331-5

[17] Larruquert J.I., Aznarez J.A., Mendez J.A., Calvo-Angos J. // Appl. Optics. 2003. V. 45. N 22. P. 4566. doi 10.1364/AO.42.004566

[18] Fernández-Perea M., Aznárez J.A., Larruquert J.I., Méndez J.A., Poletto L., Garoli D., Malvezzi A.M., Giglia A., Nannarone S. // J. Appl. Phys. 2008. V. 103. P. 073501. doi 10.1063/1.2939269

[19] Kjornrattanawanich B., Windt L.D., Uspenskii A.Y., Seely F.J. // Proc. SPIE - the International Society for Optical Engineering. 2006. P. 63170. doi 10.1117/12.681952

[20] Fernández-Perea M., Vidal-Dasilva M., Aznárez J.A., Larruquert J.I., Méndez J.A.L // Proc. SPIE, Advances in X-Ray/EUV Optics and Components III. SPIE Digital Library. 2008. V. 7077. P. 1. doi 10.1117/12.795100

[21] Saini S.M., Singh N., Nautiyal T., Auluck S. // J. Appl. Phys. 2007. V. 101. Is. 3. P. 033523. doi $10.1063 / 1.2434800$

[22] Derbez G., Petrakian J.P // Appl. Optics. 1974. V. 13. N 3. P. 599. doi 10.1364/AO.13.000599

[23] Petrakian J.P // J. Opt. Soc. Am. 1972. V. 62. N 3. P. 401. doi 10.1364/JOSA.62.000401

[24] Акашев Л.А., Кононенко В.И // ТВТ. 1994. Т. 32. В. 6. C. 825. 
[25] Акашев Л.А., Кононенко В.И // Изв. АН СССР. Металлы. 1990. № 4. C. 24.

[26] Akashev L.A., Kononenko V.I // IV Bilateral Russian-German Symposium on „Physics and Chemistry of Novel Materials“. Abstracts. Ekaterinburg: Inst. of Solid State Chemistry, 1999. V. 4. P. 1.

[27] Fujiwara H. Spectroscopic Ellipsometry: Principles and Applications. John Wiley \& Sons, Ltd, 2007. 369 p.

[28] Акашев Л.А., Попов Н.А., Шевченко В.Г // ЖПС. 2020. T. 87. № 1. C. 154. doi 10.1007/s10812-020-00974-8

[29] Климов В.В. Наноплазмоника. М.: Физматлит, 2009. 480 с.

[30] Акашев Л.А. Попов Н.А., Шевченко В.Г // ЖПС. 2018. T. 85. № 4. C. 570. doi 10.1007/s10812-018-0696-3

[31] Савицкий Е.М., Терехова В.Ф. Металловедение редкоземельных металлов. М.: Наука, 1975. 271 с.

[32] Гюнтеродт Г.И., Хаузер Э., Кюнци Х.У // Жидкие металлы. Материалы III Международной конференции по жидким металлам. М.: Металлургия, 1980. С. 176.

[33] Носков М.М. Оптические и магнетооптические свойства металлов. Свердловск: УНЦ АН СССР, 1983. 220 с.

[34] Носков М.М. Оптические свойства некоторых переходных металлов и двухполосная модель проводимости. Препринт института физики металлов УНЦ АН СССР. Свердловск, 1969. 